\title{
MuCool Superconducting Solenoid Quench Simulations and Test Stand at FNAL
}

\author{
Vladimir Kashikhin, Alan Bross, Ruben Carcagno, Darryl Orris, Daniele Turrioni
}

\begin{abstract}
MuCool superconducting solenoid fabrication and preparation for tests are in progress now. The solenoid passive quench protection system based on cold diodes and shunt resistors. The solenoid does not have heaters and quench propagation relied on the superconductor quench, and "quench back" effect caused by eddy currents in the Al coil mandrel. The solenoid has a very large inductance $\sim 600 \mathrm{H}$ and stored energy which is $100 \%$ dissipated in the cold mass during a quench. This makes their protection a challenging task. The paper presents the quench analysis of this solenoid based on 3D FEA solution of coupled transient electromagnetic and thermal problems. The simulations used the Vector Fields QUENCH code. To confirm the solenoid design at FNAL is built the Solenoid Test Stand. One of the critical issues is the solenoid fringe field which interacts with the surrounding equipment. In the paper presented field analysis and corresponding magnetic forces. The Test Stand includes the sophisticated DAQ system to monitor carefully quench events, and temperatures.
\end{abstract}

Index Terms - Superconducting solenoid, Magnetic field, Quench, 3D simulations, Test Stand.

\section{INTRODUCTION}

$\mathrm{T}$ HE MUCoOL experiment [1] magnet system is based on the superconducting solenoid designed and built by MICE collaboration [2]. The same type of superconducting solenoids should be fabricated and tested in US before the delivery to RAL for the MICE experiment.

The solenoid design [3] explores technical solutions widely used for MRI solenoids: conduction cooling by cryocoolers, HTS current leads, passive quench protection system, efficient cold mass supports, etc. Nevertheless, large magnetic field and stored energy push these solenoids close to critical parameters. Several issues were observed during MICE Spectrometer Solenoid a test [4] which has the same passive quench protection system. To reduce the risk of possible future failures, it was decided to make the solenoid quench performance analysis with 3D simulations by the commercial OPERA3D QUENCH code [5]. This code calculates the 3D transient magnetic field (code ELEKTRA) coupled with the 3D thermal analysis (code TEMPO). The main reason of using these codes is the complicated response of multi-coil magnet system where the quench propagation depends on the coupled thermal-electromagnetic effects. The superconductor and cold

Manuscript received 8 September 2012.

V. S. Kashikhin, A. Bross, R. Carcagno, D. Turrioni are with the Fermi National Accelerator Laboratory, Batavia, IL 60510 USA (corresponding author phone: 630-840-2899; fax: 630-840-6766; e-mail: kash@ fnal.gov). diodes properties used in the simulations were tested at the FNAL Superconductor Test Facility. Before the final solenoid assembly in the cryostat the solenoid cold mass will be tested in FNAL to train the solenoid at the specially built Test Stand.

\section{SUPERCONDUCTING SOLENOID}

The superconducting solenoid consists of eight coils wound on the slotted aluminum 6061 bobbin (See Fig. 1). Each coil has $1 \mathrm{~mm}$ thick ground insulation on the outer surfaces, and $3.2 \mathrm{~mm}$ on both sides. To avoid coil separation from the mandrel under Lorentz forces, the $\mathrm{Al}$ bandage is wound on top of each coil under the tension. The Al bandage ring with welded cooling tubes is mounted on the top of the solenoid cold mass assembly. The solenoid cold mass will be cooled by cryocoolers when mounted in the cryostat. For the solenoid tests and training the cold mass will be placed into the Test Stand vacuum vessel described below.

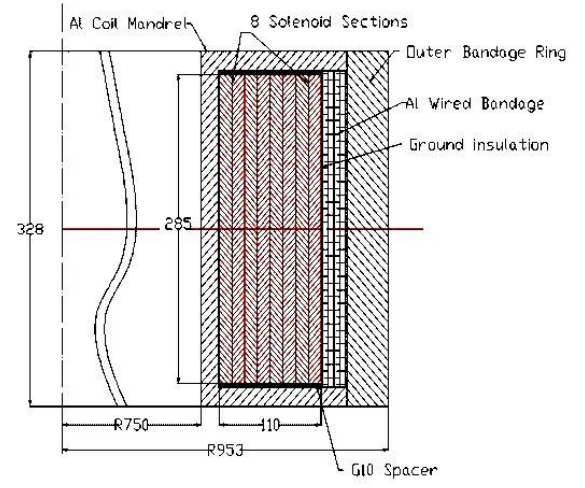

Fig. 1. Solenoid cross-section. All dimensions are in $\mathrm{mm}$.

\section{A. Solenoid Geometry and Parameters}

For the MuCool experiment was fabricated one superconducting solenoid. The solenoid electrical scheme and parameters which were used in the simulations are shown in Fig. 2 and Table 1.

TABLE I SOLENOID PARAMETERS

\begin{tabular}{lcc}
\hline \hline \multicolumn{1}{c}{ Parameter } & Unit & Value \\
\hline Peak operating current & $\mathrm{A}$ & 210 \\
Coil peak field & $\mathrm{T}$ & 7.3 \\
Solenoid coil length & $\mathrm{mm}$ & 285 \\
Bore radius & $\mathrm{mm}$ & 750 \\
Number of coil sections & & 8 \\
Total number of turns & & 15936 \\
\hline \hline
\end{tabular}




\begin{tabular}{lcc}
\hline \hline NbTi superconductor dimensions & $\mathrm{mm}$ & $0.955 \times 1.6$ \\
Cu:Sc ratio & & 4.0 \\
Critical current at $5 \mathrm{~T}, 4.2 \mathrm{~K}$ & $\mathrm{~A}$ & 760 \\
Cu RRR & & 70 \\
Inductance & $\mathrm{H}$ & 592 \\
Total stored energy & $\mathrm{MJ}$ & 13 \\
\hline \hline
\end{tabular}

\section{B. Solenoid Model for Quench Simulations}

Quench analysis of most superconducting magnet systems followed approach described in [6]. The role of "quench back" effect for the MICE solenoid was investigated in [7]. The simulations were based on predetermined from other solenoids experimental data quench propagation velocities. These velocities is strongly correlated with coil properties, and the Al mandrel heating by eddy currents induced during the quench. The 3D QUENCH code [5] is capable to simulate the more realistic quench propagation, and the influence of eddy currents on the "quench back" effect. The electrical circuit and the solenoid geometry used in the simulations are shown in Fig. 2 and Fig. 3.

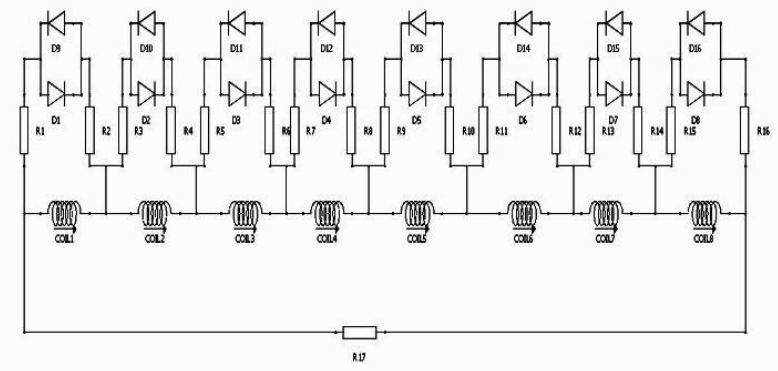

Fig. 2. Solenoid electrical circuits. D1-D16 - cold diodes, R1-R16 - $10 \mathrm{~m} \Omega$ shunt resistors, $\mathrm{R} 17-5 \Omega$ external resistor.

The solenoid is protected by a passive quench protection system formed by 16 cold diodes, and 17 shunt resistors. During quench, for example, in the Coil 1, the normal zone propagates in this coil with the corresponding resistance grows. As soon as the coil voltage reaches $4 \mathrm{~V}$ the cold diodes open, and the Coil1 current decays, flowing through R1-R2 shunt resistors. These resistors limit the coil voltage and partly reduce the losses dissipated in the coil. Because all eight coils are mounted in the radial direction close to each other, there is a strong magnetic coupling through mutual inductances. Because of the solenoid current decay, in the mandrel induced eddy currents which heat the aluminum causing the quench in the adjacent coil.

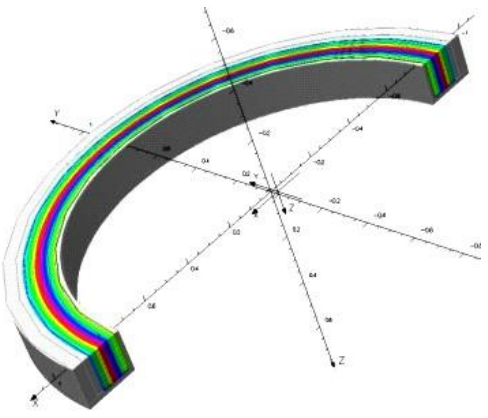

Fig. 3. Solenoid geometry used in simulations.

\section{Quench Simulation at 210 A Current}

The circuit shown in Fig. 2 was used for the quench simulations. Each coil in this circuit is protected by cold diodes and shunt resistors. External circuit is presented by 5 $\Omega$ resistor. The solenoid peak field is at the inner radius in the coil center. The quench in this point during simulation was initiated by a spot heater energized for only $0.2 \mathrm{~s}$. In $21 \mathrm{~s}$ all currents decay to $1 \%$ of the initial $210 \mathrm{~A}$ current (See Fig. 4). At the same time, the coil normal zone resistances rises up to $30 \Omega$ (See Fig. 5), and the peak coil hot spot temperature reaches $105 \mathrm{~K}$ (See Fig. 6).

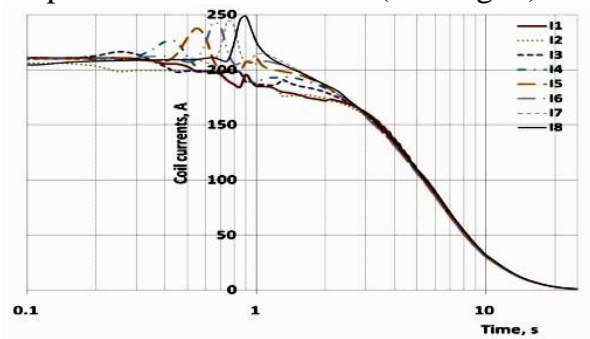

Fig. 4. Coil currents at the $210 \mathrm{~A}$ initial quench current.

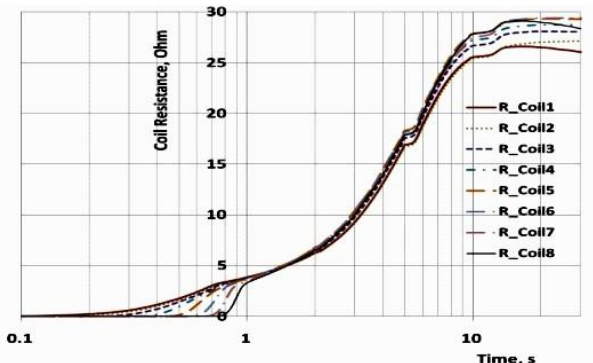

Fig. 5. Coil normal zone resistances at the $210 \mathrm{~A}$ initial quench current.

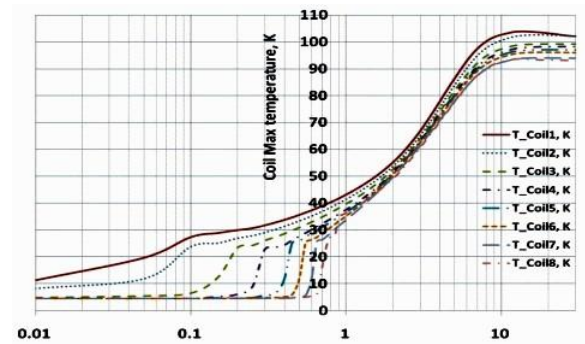

Fig. 6. Coil hot spot temperatures at the $210 \mathrm{~A}$ initial quench current.

All the above parameters look reasonable. It confirms that at peak currents, the passive quench protection system works in an agreement with the expected performance. Nevertheless, after the Spectrometer Solenoid 2B test, there was noticed a substantial shunt resistor overheating [4]. This could be explained by rather long circulating currents at the low main solenoid current quench. Fig. 7 shows the currents circulating through shunt resistors and corresponding diodes. It is obvious, that R1 current is the same as in R2 and D9, etc. The most critical parameter is the overheating of NbTi superconductor up to the level of irreversible degradation. For the used superconductor and 210 A current the critical time is $7 \mathrm{~s}$. During this time the quenched superconductor will be heated to $550 \mathrm{C}^{\circ}$ and degrades $45 \%$ [8]. Fig. 7 also shows a sharp drop of the external current through R17 $(5 \Omega)$ external resistor after 
$2 \mathrm{~s}$ of the quench. It happen when all diodes open and the main path for all sections discharge goes through inner 10 $\mathrm{m} \Omega$ resistors. It should be noted that $5 \mathrm{Ohm}$ external resistor at first moment of the quench will see $\sim 1 \mathrm{kV}$ voltage rise. It is possible to reduce this voltage by reducing this resistor value. But the variant with the $0.5 \Omega$ external resistor showed slow up to $30 \mathrm{~s}$ current discharge through this resistor and HTS current leads. So, there is some trade in and this parameter should be carefully optimized to reduce HTS leads extra heating.

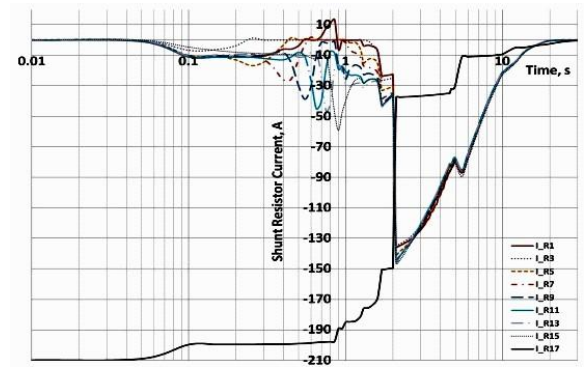

Fig. 7. Shunt resistor currents at the 210 A quench.

The quench analysis at only 100 A current showed no substantial delay in the quench propagation. For this short solenoid the low current quenches are not as critical as was observed in the long Spectrometer Solenoid [8].

\section{SUPERCONDUCTOR AND COLD Diodes TeST}

The solenoid superconductor and cold diodes used in the passive quench protection system were tested at FNAL Superconductor Test Facility. The test results were used for simulations.

\section{A. Superconductor Test Results}

Fig. 8 shows the test data of used NbTi superconductor. The peak operational current $210 \mathrm{~A}$ and the peak coil field 7.3 $\mathrm{T}$ are very close to the short sample limit $250 \mathrm{~A}$ at the operational temperature $4.5 \mathrm{~K}$.

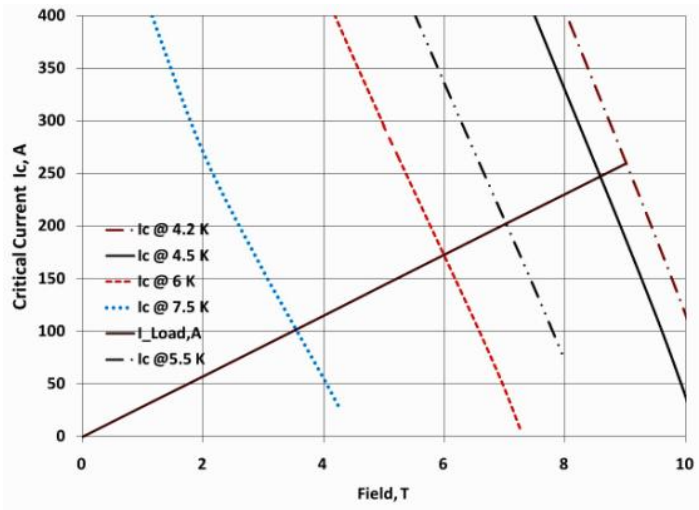

Fig. 8. Superconductor test results.

\section{B. Cold Diode Test Results}

Two R620 POWEREX diodes were tested at $4.5 \mathrm{~K}$ temperature inside Teslatron solenoid. The test setup shown in Fig. 9. There was found a strong dependence of diode forward opening voltage from the field direction and value when the direction of diode current was perpendicular to the applied magnetic field (See Fig. 10) and [9] .

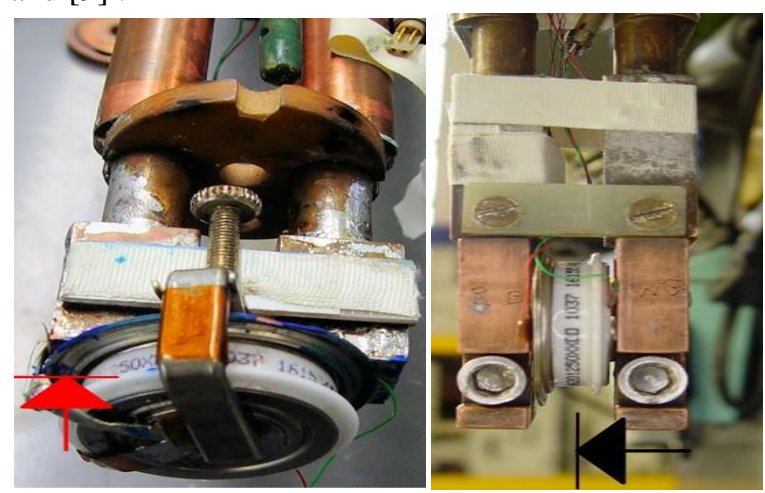

Fig. 9. Cold diodes test setup: current parallel to the field (left), and perpendicular (right).

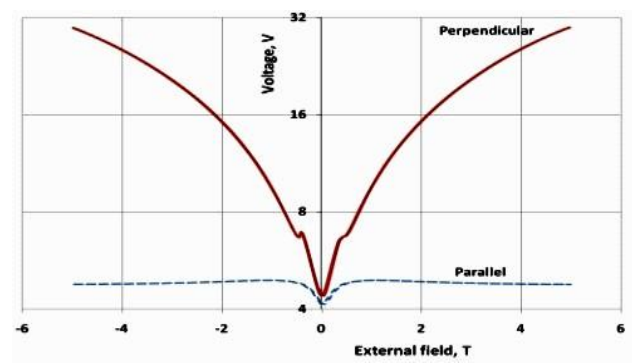

Fig. 10. Measured cold diodes opening voltages at different external fields.

In the MuCool solenoid the diode current will be in general parallel to the $2 \mathrm{~T}$ field in this area. So, for most simulations were chosen $4 \mathrm{~V}$ for the diode forward opening voltage. The simulation with $15 \mathrm{~V}$ for the perpendicular orientation did not show any noticeable effect on the quench parameters.

\section{THE FNAL TEST STAND}

The MuCool solenoid will be tested in a new test facility specially designed for testing magnets with large fringe fields [11]. This facility resides inside the FNAL Central Helium Liquefier (CHL) building where a test stand cryogenic system has been designed to interface the test cryostat with the CHL cryogenic system. Liquid helium and gaseous helium for controlled cool-down, normal operations, and controlled warm-up are supplied to the cryostat by the Fermilab Central Helium Liquefier

An integrated data acquisition and controls system was designed and built for this test stand [11]. It is made up from two primary sub-systems: The DAQ system and the Cryo Slow Scan system. The DAQ system provides quench detection/protection and data logging and is based on a system recently designed for testing the MICE Spectrometer solenoid at Wang NMR [12]. The active quench system is based on National Instrument's Compact-RIO (C-RIO), which uses a real-time operating system and FPGA. Data loggers run concurrently in both slow logger and fast logger modes, allowing data to be logged continuously at a low sample rate while remaining armed to capture a window of fast data in the event it gets a quench trigger. 
The cryo/slow scan system, which is also based on National Instrument's Compact-RIO, includes several high-resolution scans for cryogenic sensor readouts and magnet sensor measurements. This system also provides PID control loops for cryogenic valve operation.

The test coil cold mass will be placed in a large vacuum vessel [10], which was installed in CHL for this purpose. One of the issues associated with testing solenoids with large fringe fields is the Lorentz forces exerted on the solenoid from ferromagnetic objects. In order to understand the forces involved, the surrounding equipment and walls were modeled by TOSCA code [5] using corresponding iron filling factors (See Fig. 11).

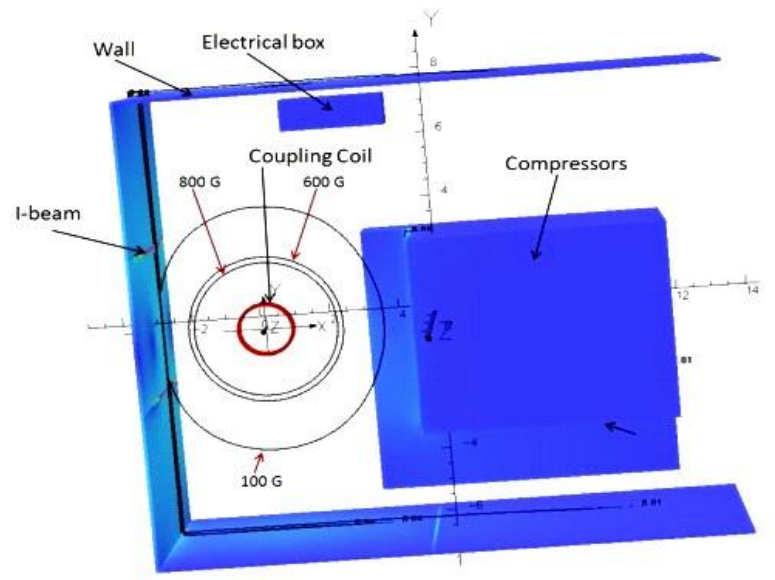

Fig. 11. The Solenoid fringing field inside CHL building.

The total Lorentz force components on the solenoid at $210 \mathrm{~A}$ current are: $\mathrm{Fx}=-260 \mathrm{~N}, \mathrm{Fy}=-100 \mathrm{~N}, \mathrm{Fz}=650 \mathrm{~N}$, and torques $\mathrm{Mx}=80 \mathrm{~N}-\mathrm{m}, \mathrm{My}=-6.1 \mathrm{kN}-\mathrm{m}$. The force components are relatively low but the torque My rotates the coil to the center of compressor volume and the corresponding support structure was built to constrain the coil inside the vacuum vessel [10]. The cold mass will be mechanically integrated with the vacuum vessel cover assembly (See Fig. 12).

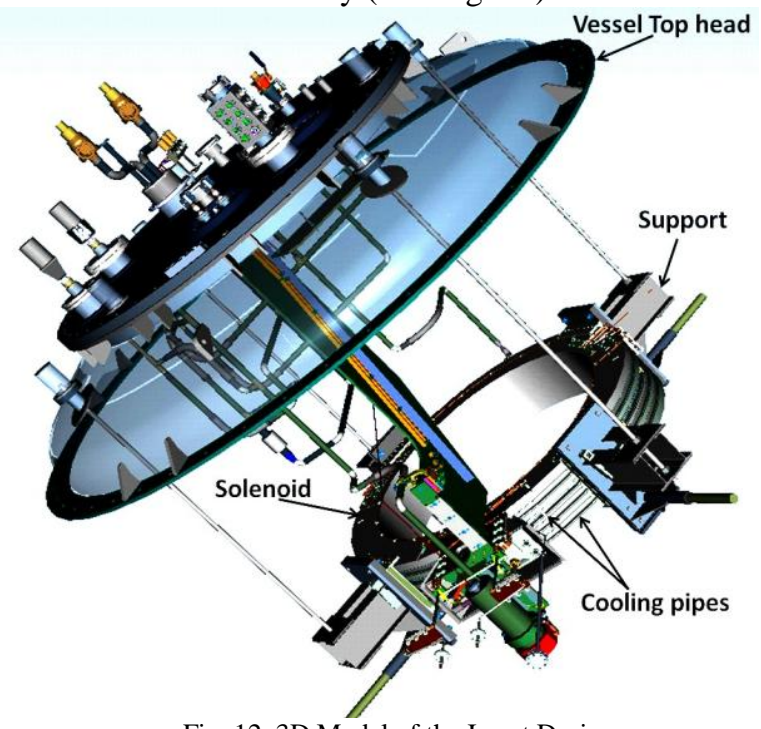

Fig. 12. 3D Model of the Insert Design.

Incorporated in the design of the structural support components mounted to the magnet are features which are designed to limit the motion of the magnet assembly in the horizontal plane during powered tests [11]. The arrangememnt of the four G-10 posts mounted inside the stainless steel sleeve is designed to carry the total estimated force of $3 \mathrm{kN}$ applied to a single rod in this direction while at the same time limiting the heat load to the magnet cold mass.

\section{CONCLUSION}

The main conclusions from the presented quench simulation results are:

1. All 8 solenoid sections quench during $\sim 1 \mathrm{~s}$ period of time at any initial current.

2. The coil current decay time for the solenoid final configuration is in the range of $20 \div 30 \mathrm{~s}$, and depends on the initial current and an external resistor.

3. The coil hot spot temperature at the 210 A quench current is $105 \mathrm{~K}$.

4. The operating current and temperature margins are low: $\Delta \mathrm{I}=40 \mathrm{~A}, \Delta \mathrm{T}=1 \mathrm{~K}$.

5. The coil leads if not heavily stabilized by copper might be overheated.

6. The solenoid quenches should be carefully monitored by DAQ system and results carefully compared with the presented results for better understanding the performance of this complicated passive quench protection system.

The MuCool solenoid cold mass will be mounted in the new cryostat and tested in the fall of 2012 .

\section{REFERENCES}

[1] "Muon Ionization Cooling R\&D", http://www.fnal.gov/projects/muon_collider/cool/cool.html

[2] G. Gregoire et al., "MICE and International Muon Ionization Experiment Technical Reference Document," http://www.mice.iit.edu, 2001.

[3] L. Wang, et al., "The Engineering Design of the $1.5 \mathrm{~m}$ Diameter Solenoid for the MICE RFCC Modules", IEEE Transactions on Applied Superconductivity, vol. 18, no. 3, 2008, p. 937.

[4] M.A. Green, S.P. Virostek, M.S. Zisman, "The Results of Recent MICE Spectrometer Solenoid Tests," IEEE Transactions on Applied Superconductivity, vol. 21, no. 3, 2011, pp. 1764-1767.

[5] "Opera-3d User Guide," VF-07-11-B3, Cobham Technical Services, Vector Fields Software, Oxford, England, p. 523.

[6] M.N. Wilson, "Superconducting Magnets," Oxford University Press, Oxford, 1983, p. 330.

[7] X. L. Guo, et al., "Quench Protection for the MICE Cooling Channel Coupling Magnet", IEEE Transactions on Applied Superconductivity, vol. 19, no. 3, 2009, pp. 1360-1363.

[8] V. Kashikhin, A. Bross, S. Prestemon, "Quench Analysis of MICE Spectrometer Superconducting Solenoid", IEEE Transactions on Applied Superconductivity, vol. 22, no. 3, 2012, p. 4702904.

[9] S. Yamamoto, et al., "Quench Protection of Persistent Current Switches using Diodes in Cryogenic Temperature", Power Electronics Specialists Conference, PESC'88, $19^{\text {th }}$ Annual IEEE, vol.1, 1988, pp. 321 -325.

[10] R. D. Luther, "Design and Fabrication of a Large Cryostat for Testing SMES Apparatus", Advances in Cryogenic Engineering, Vol. 45, 2000, pp. 1699-1705.

[11] "MICE CC Test Stand Technical Design Report", http://tiweb.fnal.gov/website/controller/2184

[12] R. Pilipenko, R. H. Carcagno, A. Makulski, R. Nehring, D. F. Orris, "An FPGA-Based Quench Detection and Continuous Logging System for Testing Superconducting Magnets," IEEE Trans. Appl. Supercond. submitted for publication. 